\title{
Variabilidad y confiabilidad de los tiempos registrados de procesos operatorios de cesáreas realizadas en una clínica particular de Lima, Perú, 2011
}

Variability and reliability of times registered for cesarean delivery surgical processes at a private clinic, Lima, Peru, 2011

\author{
Manuel André Virú-Loza ${ }^{1,2, a}$, Carlos Enrique León-Torres ${ }^{1, a}$, Sergio José Flores- \\ Castañeda $^{1, a}$, Gerardo Arnaldo Trelles-Bustamante ${ }^{1, a}$, Juan José Montenegro-Idrogo ${ }^{1,3, a}$ \\ ${ }^{1}$ Facultad de Medicina Humana, Universidad Nacional Mayor de San Marcos, Lima, Perú. \\ ${ }^{2}$ Asociación para el Desarrollo de la Investigación Estudiantil en Ciencias de la Salud, Universidad Nacional Mayor de San Marcos (ADIECS- \\ UNMSM), Lima, Perú. \\ ${ }^{3}$ Sociedad Cientifica San Fernando, Universidad Nacional Mayor de San Marcos, Lima, Perú.
}

${ }^{a}$ Estudiante de medicina

\begin{abstract}
Resumen
Introducción: La evaluación de la eficiencia es una herramienta útil para una correcta toma de decisiones en los centros de salud. Esta incluye conocer la variabilidad y confiabilidad de los tiempos de procedimientos médicos y quirúrgicos registrados. Objetivos: Determinar la variabilidad y confiabilidad de los tiempos registrados de procesos operatorios de cesáreas. Diseño: Estudio transversal. Institución: Clínica Centenario Peruano Japonesa. Participantes: Registros de pacientes con cesáreas. Intervenciones: A partir del registro operatorio y del registro de las historias clínicas se obtuvo datos médicos y de tiempos operatorios, durante el año 2011. Principales medidas de resultados: Medidas de dispersión de todos los indicadores y coeficientes de correlación intraclases (CCIs) entre los registros evaluados. Resultados: El tiempo de traslado del paciente a sala de operaciones presentó la mayor variabilidad y los CCls entre los registros utilizados con respecto al tiempo de inicio y finalización de las cesáreas fueron 0,902 y 0,901, respectivamente. Conclusiones: En la clínica estudiada, el tiempo de traslado del paciente a sala de operaciones fue el más variable, existiendo un buen grado de confiabilidad entre los registros estudiados. Los análisis de eficiencia y productividad para mejorar procesos quirúrgicos en nuestro medio deben ser publicados para que sirvan de utilidad en otros centros de salud.
\end{abstract}

Palabras clave: Variabilidad, confiabilidad, tiempos operatorios, cesárea.

\section{Abstract}

Introduction: Efficiency evaluation is a useful tool for making right decisions at health care institutions. This includes knowing the variability and reliability of registered medical and surgical procedures times. Objectives: To determine variability and confidence of registered cesarean deliveries surgical processes times. Design: Cross-sectional study. Institution: Clinica Centenario Peruano Japonesa. Participants: Patients' cesarean deliveries registries. Interventions: Medical and surgical times data were obtained from the surgical registry and clinical records' registry, 2011. Main outcome measures: Dispersion measures of all indicators and intraclass correlation coefficients (ICCS) from the studied registries. Results: Operating room patient transfer time showed the greatest variability and the ICCs between studied registries for starting and ending time of cesarean delivery were 0.902 and 0.901 , respectively. Conclusions: At the private clinic studied patient transfer to the operating room was the most varied, and there was good reliability between operatory registries and clinical records' registries. Efficiency and productivity analyses to improve surgical processes need to be published in order to be useful to other health care centers.

Key words: Variability, reliability, surgical times, cesarean delivery.

An Fac med. 2013;74(1):37-41

\section{INTRODUCCIÓN}

Según las "Normas técnicas para proyectos de arquitectura y equipamiento de las unidades de centro quirúrgico" del Ministerio de Salud del Perú MINSA, un centro quirúrgico es definido como un conjunto de ambientes cuya función gira alrededor de las salas de operaciones y que proporciona al equipo quirúrgico las facilidades ne- cesarias para efectuar procedimientos quirúrgicos en forma eficaz, eficiente y en condiciones de máxima seguridad con respecto a contaminaciones ${ }^{(1)}$.

La evaluación de la calidad de los servicios de operaciones reflejada en la eficiencia del uso de sala es una herramienta útil para mantener parámetros respecto a tiempos útiles y seguros que permitan una correcta toma de deci- siones en la gestión de recursos y programaciones realizadas en estos centros hospitalarios, la cual es aplicada a establecimientos públicos y privados ${ }^{(2)}$. Ello supone un proceso continuo y asociado a diversos factores, como son los recursos humanos (personal calificado, disponible y suficiente), insumos o materiales y la complejidad y factibilidad de los procesos que requieren y justifican una intervención oportuna ${ }^{(3)}$. 
La evaluación de la eficiencia se hace en relación a un mayor rendimiento en producción de servicios de salud obtenidos a un menor costo social posible ${ }^{(4)}$, además de la importancia de los tiempos efectivos de uso de sala de operaciones, como otros indicadores efectivos en relación a los procesos pre, peri y postoperatorios ${ }^{(5-7)}$.

Sin embargo, el proceso de control se encuentra limitado al subregistro en la mayoría de centros, y se menciona que el personal, la falta de apoyo logístico, el ambiente inadecuado de abordaje al paciente y la información familiar insuficiente se convierten en factores importantes del mal registro de historias clínicas en la evaluación de calidad ${ }^{(8)}$. Estos aspectos se asocian a complicaciones que generan costos mayores, en los cuales el manejo puede estar limitado a la cobertura de atención que presente el paciente (asegurado o particular), situaciones que difieren entre centros de referencia públicos y privados ${ }^{(9,10)}$, siendo necesaria la búsqueda de soluciones ante esta problemática ${ }^{(11)}$.

Algunos autores proponen indicadores, como exceso de costo del recursos humanos, tardanza de hora de inicio, tasa de reprogramaciones, tiempos de admisión en la Unidad de Cuidados Postanestésicos, margen de contribución promedio por hora de sala de cirugía, tiempo de rotación o recambio, predicción de sesgo y/o prolongación de tiempos quirúrgicos ${ }^{(12)}$, así como oportunidad para urgencias y rentabilidad bruta ${ }^{(5,13)}$. Uno de estos parámetros es conocido como gap time, el que se operacionaliza como el intervalo desde el tiempo de programación y el inicio real de la operación. Esto permite evaluar la capacidad de acomodación de un evento no planificado (emergencia) y se asocia directamente a tiempos prolongados de estancia postoperatoria ${ }^{(6,7)}$.

En este escenario, uno de los aspectos que cobra importancia evaluar debido a su frecuencia e implicancias en cuidados y consecuencias que forman parte de los lineamientos en salud de los Objetivos del Desarrollo del Mile- nio (mortalidad materna e infantil), son los procesos ginecoobstétricos y en particular las cesáreas. Se menciona que estas han ido en aumento, siendo ascendente el número de cesáreas electivas. No obstante, se señala factores asociados a este tipo de presentación, como determinantes socioeconómicos, culturales y de difusión de información, la autonomía del paciente en la toma de decisiones, procesos tecnológicos, así también otros de tipo ético-legal y de posibles complicaciones asociadas; además, el tipo de seguro ${ }^{(14)}$.

Para lograr un panorama general de los tiempos en que se realiza procedimientos relacionados a cesáreas, el presente estudio tuvo por objetivo evaluar la variabilidad y confiabilidad de los tiempos registrados de procesos operatorios correspondientes a cesáreas realizadas en una clínica particular en Lima, Perú, durante el año 2011.

\section{MÉTODOS}

Se realizó un estudio transversal, entre los meses de enero y diciembre de 2011. Para esto, previamente se obtuvo aprobación de la dirección de la clínica en la que se realizó el estudio, para la revisión de los libros de registro operatorio e historias clínicas.

Nuestra población objetivo consistió en todos los registros de cesárea consignados en los registros operatorios de la Clínica Centenario Peruano Japonesa, en el período enero a diciembre de 2011.

Trabajamos con una muestra aleatoria simple correspondiente a $40 \%$ de los casos de nuestra población objetivo, constituyendo esto un total de 85 casos analizados. Este porcentaje se debe al número máximo que logísticamente se nos permitió analizar en el plazo que teníamos establecido.

Se realizó la revisión del registro operatorio y de las historias clínicas en búsqueda de los siguientes indicadores: edad de la gestante, edad gestacional, gestación previa, cesárea previa, diag- nósticos preoperatorios, tiempo entre el inicio de la anestesia y el inicio de la cesárea en minutos, tiempo entre el inicio y la finalización de cesárea según registro operatorio en minutos, tiempo entre el inicio y la finalización de cesárea según historia clínica en minutos y, finalmente, el tiempo entre la finalización de la cesárea y la finalización del periodo anestésico en minutos.

En el análisis estadístico, primero se calculó medidas de resumen de todos los indicadores mencionados: se obtuvo las frecuencias para variables categóricas, y medidas de tendencia central y dispersión para las numéricas. Luego, se utilizó la prueba de KolmogorovSmirnov para verificar la normalidad de los distintos datos obtenidos. Posterior a esto se calculó el coeficiente de correlación intraclases utilizando los tiempos de inicio y fin de la cesárea que se encontraban consignados en las fuentes de información mencionadas, con la finalidad de evaluar la confiabilidad de los tiempos de procesos operatorios consignados en los dos registros señalados.

Se realizó los análisis estadísticos con el paquete estadístico SPSS v.20.0.

\section{RESULTADOS}

Las medidas de resumen correspondientes a las variables estudiadas están descritas en la tabla 1.

En cuanto a los tiempos de los procesos operatorios, podemos observar a través de gráficos box-plot en la figura 1 la existencia de varios casos en los que se excede demasiado el tiempo en que normalmente se realizan dichos procesos.

A partir de los resultados mostrados en la tabla 1 y figura 1 , podemos observar que el tiempo de traslado del paciente a sala de operaciones tuvo la mayor variabilidad.

Previo a la realización de las pruebas de confiabilidad, primero se analizó la normalidad de los datos a utilizar para 
Tabla 1. Datos descriptivos de las cesáreas realizadas en la Clínica Centenario Peruano Japonesa, 2011.

\begin{tabular}{|c|c|}
\hline Variables & $\begin{array}{l}\text { Medidas de } \\
\text { resumen }\end{array}$ \\
\hline Edad de la gestante & $31,2 \pm 5,2^{*}$ \\
\hline Edad gestacional & $38,1 \pm 1,9^{*}$ \\
\hline Gestación previa & $41(48,2)^{* *}$ \\
\hline Cesárea previa & $21(24,7)^{* *}$ \\
\hline \multicolumn{2}{|l|}{ Diagnósticos CIE10 } \\
\hline Edema, proteinuria e hipertensión en embarazo, parto y puerperio (010 - 016) & $6(7,1)^{* *}$ \\
\hline Complicaciones del embarazo que requieren una atención a la madre (030 - 048) & $60(70,6)^{* *}$ \\
\hline Complicaciones del embarazo y el parto (082.0) & $34(40)^{* *}$ \\
\hline Parto por cesárea electiva & $1(1,2)^{* *}$ \\
\hline Otras enfermedades (095 - 099) & $6(7,1)^{* *}$ \\
\hline \multicolumn{2}{|l|}{ Tiempos de procesos operatorios (minutos) } \\
\hline Tiempo de traslado del paciente a sala de operaciones & $20[15-35]^{* * *}$ \\
\hline Tiempo entre inicio de anestesia e inicio de la cesárea & $15[10-20]^{* * *}$ \\
\hline Tiempo entre inicio y finalización de cesárea según registro operatorio & $42,89 \pm 15,39 *$ \\
\hline Tiempo entre inicio y finalización de cesárea según historia clínica & $43,01 \pm 12,13^{*}$ \\
\hline Tiempo entre finalización de cesárea y finalización de periodo anestésico & $9[5-10]^{* * *}$ \\
\hline
\end{tabular}

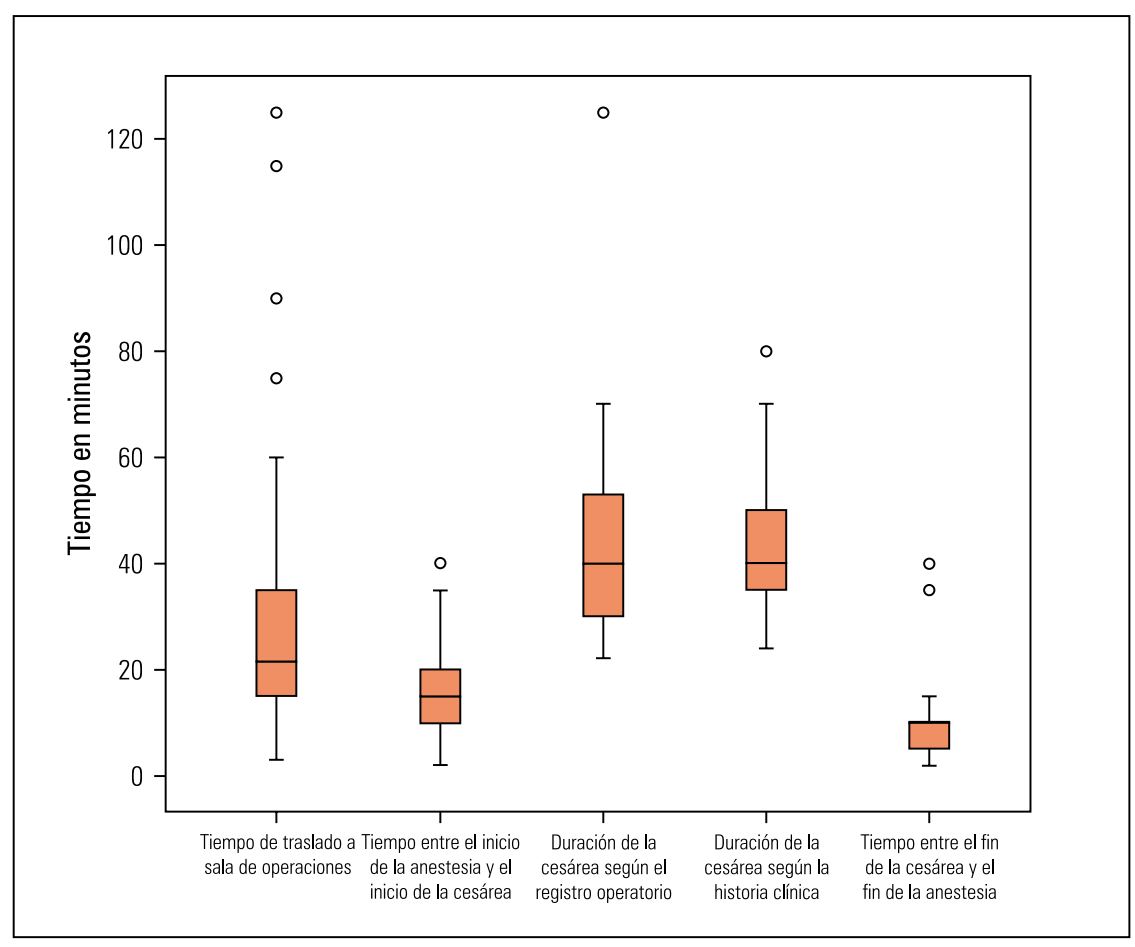

Figura 1. Duración registrada en minutos de procesos operatorios de cesáreas. estos fines. Todos los indicadores cuantitativos de inicio y finalización de cesárea en ambas fuentes de información demostraron tener distribución normal, al utilizar la prueba de KolmogorovSmirnov (resultados no mostrados).

Teniendo en cuenta la distribución normal de los datos, se utilizó el coeficiente de correlación intraclases (CCI) para medidas promedio, utilizando un modelo de efectos aleatorios de dos vías. Esta prueba estadística fue aplicada en los datos de tiempo de inicio de cesáreas registrados en el libro de registro operatorio y en las historias clínicas, resultando un valor de $\mathrm{CCI}=0,902$; el mismo análisis estadístico aplicado a los datos de tiempo de finalización de cesáreas provenientes de los registros mencionados produjo un $\mathrm{CCI}=0,901$.

\section{DISCUSIÓN}

El tiempo de traslado a sala de operaciones tuvo la mayor variabilidad comparado con los demás indicadores. Por otro lado, si observamos los gráficos boxplot, igualmente podemos evidenciar que este tiempo de traslado presentó la mayor cantidad de datos atípicos. Esto nos indica que la mayor disparidad en cuanto a tiempos de ejecución de actos protocolares en cesáreas programadas la tiene el indicador mencionado. Esta desviación estándar es importante si se la compara con las medidas descriptivas de los otros indicadores de procesos descritos, principalmente el tiempo de realización de las cesáreas, ya que visto como una serie de procesos llevados a cabo uno tras otro a lo largo de un día, una reducción de la cantidad de procesos de traslado lejanos del promedio significaría una oportunidad de programar más cesáreas (o al menos una sola más) durante el transcurso del día. Entonces, al ser 22,48 minutos una desviación considerable como para tener una repercusión en la capacidad operativa diaria de la clínica -en lo que concierne a cesáreas programadas- es importante abordar los problemas logísticos en este punto. 
La reducción del tiempo de determinados procesos factibles de ser reducidos es algo que constituye una estrategia para mejorar la eficiencia de un centro de salud. Ejemplo de esta premisa son los estudios en los que se demuestra una mejor eficiencia y mejores ganancias cuando el tiempo de estadía de los pacientes es el mínimo posible ${ }^{(15)}$ y cuando el tiempo en que las salas de operaciones se encuentran sin actividad se reduce al mínimo ${ }^{(7)}$.

La variación propiamente dicha de los tiempos operatorios puede deberse a distintas clases de problemas logísticos, entre los que puede incluirse interrupciones, fallas de comunicación entre el personal involucrado, familiaridad entre miembros del equipo y el factor de variación impredecible propio de cada cirugía realizada ${ }^{(16)}$. Además, otro de los factores que en otros estudios influye en la variación de los tiempos operatorios es el tipo de anestesia utilizada en distintos tipos de procedimientos quirúrgicos ${ }^{(17)}$; sin embargo, en nuestro estudio todos los procedimientos fueron del mismo tipo, utilizando el mismo tipo de anestesia. Por otro lado, un factor que se menciona como hipotético es el hecho de tener planificado un procedimiento quirúrgico y finalmente realizar otro en vez del programado ${ }^{(18)}$. Además, se ha demostrado que los factores relacionados al cirujano -como son la edad, experiencia y génerocontribuyen a mejorar los modelos de predicción de tiempos operatorios ${ }^{(19)}$. Igualmente, las características del paciente ayudan a mejorar estos modelos de predicción ${ }^{(20)}$.

Tal y como se ha mencionado previamente, estudios anteriores demuestran la importancia de mejorar los procesos que consumen tiempo potencialmente utilizable en más procedimientos en el transcurso del día, favoreciendo la eficiencia general del establecimiento de salud.

Si bien la mayor información que se tiene sobre la optimización de tiempos de procesos o procedimientos médicos proviene de estudios en establecimientos de salud estatales, esto también es aplicable a establecimientos privados, con ciertas salvedades de interpretación.

En el caso de los establecimientos estatales, nosotros nos enfrentamos a la necesidad imperiosa de realizar la mayor cantidad de procesos y/o procedimientos médicos, con la finalidad de alcanzar a atender la mayor parte de la población; por otro lado, también tenemos al mismo tiempo que abordar asuntos de administración de recursos ${ }^{(11)}$. Sin embargo, en cuanto a materia de obtención de recursos financieros existe un alto grado de dependencia -al menos en nuestro medio-de instancias gubernamentales responsables de la administración de recursos del estado.

Para los establecimientos privados está presente la intención de atender la mayor cantidad de pacientes con la finalidad de obtener recursos financieros, ya que son instituciones con un mecanismo de conseguir recursos totalmente independientes del estado.

Estas diferencias mencionadas entre establecimientos estatales y privados, en lo que respecta a la optimización de tiempos, se refleja en el hecho de tener: (a) establecimientos de salud estatales que con una cantidad estable definida de recursos suministrados por el gobierno deben atender la mayor cantidad de población; y, (b) establecimientos de salud privados cuya cantidad de recursos, estabilidad institucional, economía, oportunidad de crecimiento y permanencia en el tiempo pueden variar considerablemente de acuerdo a cuántos pacientes se logre atender.

Habiendo dicho esto, podemos decir que, en este caso particular, la optimización de los tiempos de traslado a sala de operaciones de pacientes que son sometidas a cesárea contribuiría a mejorar la eficiencia y manejo financiero de la clínica, al menos en cuanto a lo que concierne a las cesáreas.

En cuanto a la confiabilidad, podemos evidenciar que el tiempo de inicio y finalización de cesárea registrados por el personal de salud tiene un buen grado de confiabilidad, tanto para el caso del registro de inicio de cesárea como el registro de finalización de cesárea. Probablemente esto se deba a que, si bien no son los mismos profesionales los que registran los datos en los registros analizados, trabajan en una institución que está bajo constante auditoría, lo que implica un continuo control del rendimiento de los trabajadores.

En conclusión, en la clínica particular estudiada, el tiempo de traslado a sala de operaciones es el indicador con mayor variación con respecto al promedio. Por otro lado, existe un óptimo grado de confiabilidad entre el registro operatorio y las historias clínicas con respecto a las horas de inicio y finalización de las cesáreas.

Debido a la llamativa variación de los valores de tiempo de traslado a sala de operaciones y a la magnitud de esta variación con respecto a los tiempos de otros procesos, debe analizarse más de cerca este proceso. Además, debe estudiarse de igual manera otros procesos más específicos que forman parte de los protocolos de cesárea propios de la clínica, que no han sido analizados en este estudio.

También, es necesario evaluar en la clínica la concordancia de los tiempos en que se programa las cirugías con respecto a los tiempos en que realmente son realizadas y de esta manera mejorar la evaluación de indicadores de eficiencia.

Finalmente, los análisis de eficiencia y productividad para mejorar los procesos quirúrgicos que puedan ser realizados a futuro en esta clínica particular y en otros centros de salud de nuestro medio, privados o estatales, deben ser efectuados y publicados para que dicha experiencia sirva de utilidad en la realización de este tipo de análisis y formulación de estrategias en otros centros de salud.

\section{AGRADECIMIENTOS}

Al Dr. Juan Matzumura Kasano y al Mg. Hugo Gutiérrez Crespo. 


\section{REFERENCIAS BIBLIOGRÁFICAS}

1. Ministerio de Salud. Dirección General de Salud de las Personas. Normas técnicas para proyectos de arquitectura y equipamiento de las unidades de centro quirúrgico. 2000. [acceso viernes 29 de junio de 2012]. Disponible en: http://www. minsa.gob.pe/dgiem/infraestructura/WEB_DI/ NORMAS/Normas\%20Cirug\%C3\%ADa\%204\%20 MINSA.pdf

2. Ministerio de Salud. Directiva Sanitaria No MINSA DGSP-V.01 Indicadores de Salud Hospitalarios. [acceso viernes 29 de junio de 2012]. Disponible en: ftp://ftp2.minsa.gob.pe/docconsulta/documentos/dgsp/DIRECTIVASINDICADORESS $\% 20 \% 20$ HOSPITALARIOSV2105.doc

3. Cubillo YO. Rendimiento de quirófanos en los hospitales de la Caja Costarricense de Seguro Social en el año 2010. [acceso viernes 29 de junio de 2012]. Disponible en: http://portal.ccss. sa.cr/portal/page/portal/Gerencia_Administrativa/ DireccionComprasServiciosdeSalud/EstudiosRealizados/Rendimiento_de_Quirofanos_CCSS.pdf

4. Jiménez RE. Indicadores de calidad y eficiencia de Ios servicios hospitalarios: Una mirada actual. Rev Cubana Salud Pública. 2004;30(1):17-36.

5. Bejarano M. Evaluación cuantitativa de la eficiencia en las salas de cirugía. Rev Colomb Cir. 2011;26:273-84.

6. Fajardo G, Aguirre HG, Robledo H. Sistema de evaluación por monitoría para hospitales de alta especialidad. Gac Méd Méx. 2011;147:411-9.

7. Dhupar R, Evankovich J, Klune JR, Vargas LG, Hughes SJ. Delayed operating room availability significantly impacts the total hospital costs of an urgent surgical procedure. Surgery. 2011;150(2):299-305.
8. Pablo-Roncano E. Evaluación de la calidad de los registros de las historias clínicas de los pacientes fallecidos en el Servicio de Emergencia de un Hospital General. Rev Soc Peru Med Interna 2008;21(2):51-4.

9. Garcia F, Cieza J, Flores J, Huapaya J, Obregón A. Una década de altas médicas no efectivizadas en un hospital de Lima, Perú. Rev Peru Med Exp Salud Publica. 2012;29(2):228-31.

10. Thomas WJ, Guire KE, Howart GG. Is patient length of stay related to quality of care? Hosp Health Serv Admin. 1997;42(4):489-507.

11. Girotto JA, Koltz PF, Drugas G. Optimizing you operating room: or, why large, traditional hospitals don't work. Int J Surg. 2010;8(5):359-67.

12. Macario A. Are your hospital operating rooms "efficient"? A scoring system with eight performance indicators. Anesthesiology. 2006;105(2):237-40.

13. Serra Sutton V, Solans Domènech M, Espallargues Carreras M. Eficiencia en la utilización de los bloques quirúrgicos. Definición de indicadores. Plan de Calidad para el Sistema Nacional de Salud del Ministerio de Sanidad, Política Social e Igualidad. Ministerio de Ciencia e Innovación. Agència d'Informació, Avaluació i Qualitat en Salut; 2011. Informes de Evaluación de Tecnologias Sanitarias: AIAQS núm. 2010/04

14. Kizer S. Frecuencia de la cesárea. Factores resaltantes relacionados con su incremento. Gac Méd Caracas. 2011;119(1):12-21.

15. Martinussen PE, Midttun L. Day surgery and hospital efficiency: empirical analysis of Norwegian hospitals, 1999-2001. Health Policy. 2004;68(2):18396.

16. Gillespie BM, Chaboyer W, Fairweather N. Factors that influence the expected length of operation: results of a prospective study. BMJ Qual Saf. 2012;21(1):3-12.
17. Strum DP, Sampson AR, May JH, Vargas LG. Surgeon and type of anesthesia predict variability in surgical procedure times. Anesthesiology. 2000;92(5):1454-66.

18. Dexter F, Dexter EU, Ledolter J. Influence of procedure classification on process variability and parameter uncertainty of surgical case durations. Anesth Analg. 2010;110(4):1155-63.

19. Stepaniak PS, Heij C, de Vries G. Modeling and prediction of surgical procedure times. Statistica Neerlandica. 2010;64(1):1-18.

20. Eijkemans MJ, van Houdenhoven M, Nguyen T, Boersma E, Steyerberg EW, Kazemier G. Predicting the unpredictable: a new prediction model for operating room times using individual characteristics and the surgeon's estimate. Anesthesiology. 2010;112(1):41-9.

Artículo recibido el 29 de setiembre de 2012 y aceptado para publicación el 30 de noviembre de 2012.

Financiamiento:

Autofinanciado.

Correspondencia:

Manuel André Virú-Loza

Dirección: Jr. Piedra Bigua 2241-A, San Juan de Lurigancho

Teléfono: 3043417 / 993774567

Correo electrónico: andreviru@hotmail.com 